\title{
Snodar: a new instrument to measure the height of the boundary layer on the Antarctic plateau
}

\author{
Colin S. Bonner*a ${ }^{\text {a }}$ Michael C. B. Ashley ${ }^{a}$, Jon S. Lawrence ${ }^{a}$, John W. V. Storey ${ }^{a}$, \\ Daniel M. Luong-Van ${ }^{\mathrm{a}}$, Stuart G. Bradley \\ ${ }^{a}$ School of Physics, University of New South Wales, Sydney NSW 2052, Australia \\ ${ }^{\mathrm{b}}$ Faculty of Science, University of Auckland, Auckland, New Zealand
}

\begin{abstract}
The height of the atmospheric boundary layer on the Antarctic plateau is of particular importance to designers of optical telescopes for Antarctica. Snodar was developed at the University of New South Wales to measure the height of the atmospheric boundary layer at Dome A and Dome C on the Antarctic plateau. Snodar, or Surface layer Non-Doppler Acoustic Radar, is a true monostatic high-frequency acoustic radar (SODAR) operating between $5 \mathrm{kHz}$ and $15 \mathrm{kHz}$. As the height of the boundary layer at Dome $\mathrm{C}$ is expected to be less then $30 \mathrm{~m}$, and unknown at Dome A, Snodar was designed to have a minimum sampling height of $5 \mathrm{~m}$ with a vertical resolution of $1 \mathrm{~m}$ or better. Snodar uses a PC/104 computer to perform signal processing in real time, and a USB sound card for low-latency analog IO. Snodar was designed to run autonomously storing data on USB flash disks for retrieval the following summer, while uploading of data acquisition scripts and spot checking of data is possible via Iridium satellite through UNSW's PLATO facility. Snodar also incorporates a unique in-situ calibration sphere. We present details of the design and results from testing of Snodar.
\end{abstract}

Keywords: atmosphere, boundary layer, SODAR, Antarctica, Antarctic plateau, astronomy, site-testing

\section{INTRODUCTION}

The nature of the atmospheric boundary layer (ABL) over the Antarctic plateau is of interest to meteorologists, atmospheric chemists, and astronomers alike. The height, strength, and variability of the ABL is of particular importance to astronomers since it directly affects image quality. At Dome $\mathrm{C}$, the turbulence in the free atmosphere above the ABL has been found to be exceptionally calm and stable $\mathrm{e}^{1,2}$. However, the winter-time ABL, which is typically tens of metres thick, was found to be a very turbulent layer that contributes up to $87 \%$ of the winter-time astronomical "seeing" (i.e., image degradation due to the earth's atmosphere) at ground level ${ }^{2}$. At a height of $4093 \mathrm{~m}$, Dome A is the highest point on the Antarctic plateau as shown in Fig 1. While data have yet to be obtained, simulations based on atmospheric and climate models predict that the winter-time surface turbulent layer at Dome A will be weaker and confined closer to the surface than that observed at Dome $\mathrm{C}^{3}$.

In January 2008, the PLATeau Observatory (PLATO) was deployed to Dome $\mathrm{A}^{4}$ as part of the Polar Research Institute of China PANDA traverse. This project was a collaboration between the University of New South Wales, the Polar Research Institute of China, and the National Astronomical Observatories of the Chinese Academy of Sciences, with additional instrumentation being provided by institutes in the US and the UK. One of the motivations for PLATO was to investigate the ABL at Dome A. Unfortunately, no commercially available acoustic radars could probe the first few tens of meters of the atmosphere with a resolution of $1 \mathrm{~m}$ or less. While fully-instrumented towers or tethered sondes are able to provide the data required, they are either too expensive, require a large amount of logistics or are unable to be operated autonomously. Snodar, or Surface-layer Non-Doppler Acoustic Radar, is a new instrument designed specifically to autonomously measure the height, strength, and variability of the ABL on the Antarctic plateau. We present here technical details of the design and construction of Snodar along with initial results from testing the instrument.

*Email: cbonner@phys.unsw.edu.au

Ground-based and Airborne Instrumentation for Astronomy II, edited by lan S. McLean, Mark M. Casali, Proc. of SPIE Vol. 7014, 70146I, (2008) · 0277-786X/08/\$18 · doi: 10.1117/12.788154 


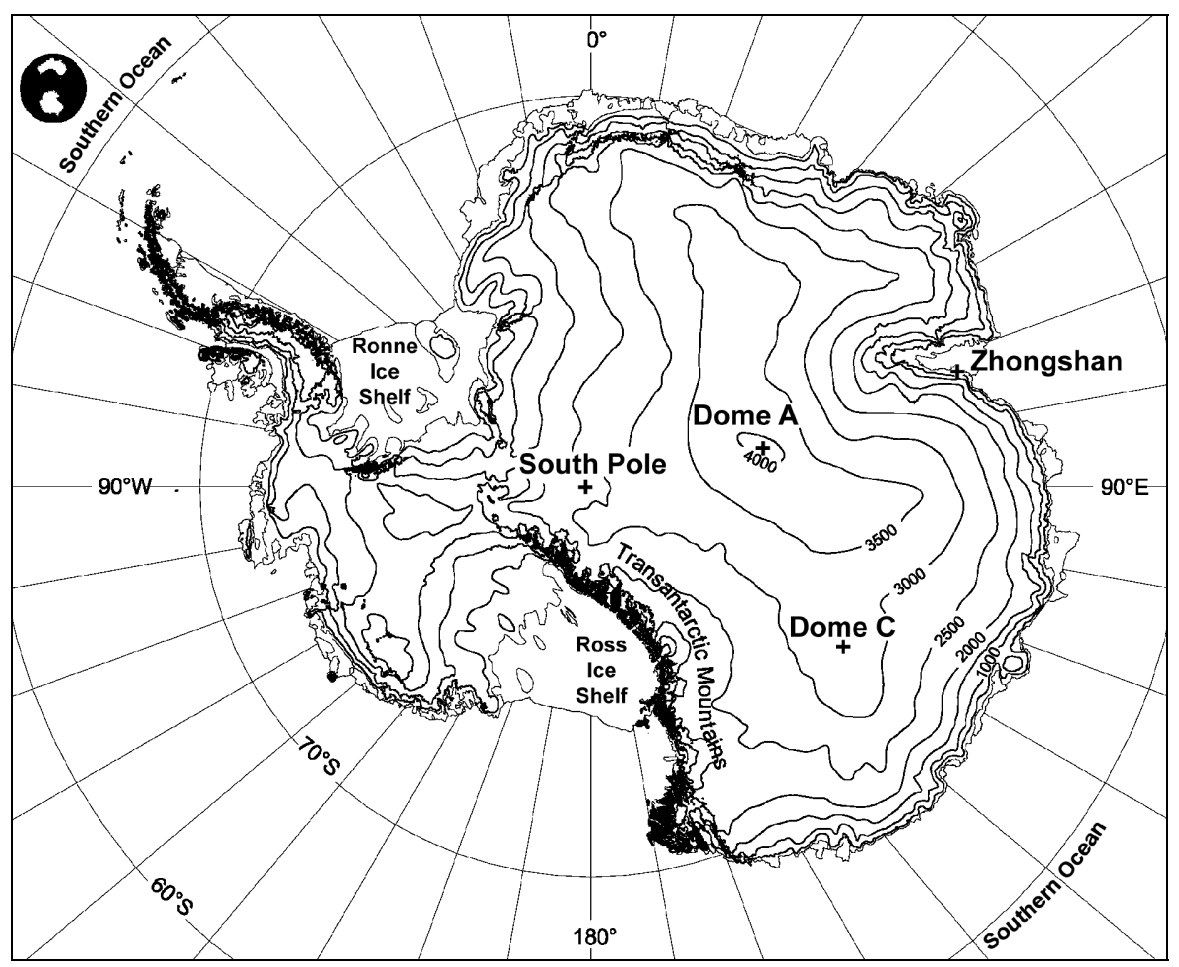

Fig 1 Map of Antarctica showing the Chinese coastal station Zhongshan and the high plateau stations South Pole, Dome C and Dome A. Basic map courtesy of the Australian Antarctic Data Centre.

\section{SNODAR}

Snodar's primary design goal was to measure the atmospheric temperature structure function, $C_{T}^{2}$, from a height of $5 \mathrm{~m}$ up to at least $100 \mathrm{~m}$ with a resolution of $1 \mathrm{~m}$ or better. In 1969, Little ${ }^{5}$ demonstrated the principle and feasibility of measuring the temperature structure of the atmosphere with an acoustic radar. Snodar uses this concept, sending an intense acoustic pulse into the atmosphere and then listens for the faint scattering from temperature inhomogeneities. In order to sample the atmospheric temperature structure to a resolution of $1 \mathrm{~m}$ or better the acoustic pulse must be $2 \mathrm{~m}$ in length or shorter ${ }^{6}$. This equates to a pulse length of $6.6 \mathrm{~ms}$ at an air temperature of $-40^{\circ} \mathrm{C}$.

Snodar must operate autonomously throughout the Antarctic winter with low bandwidth communications and without the possibility of human intervention at the Dome A site. To increase reliability we implemented as much of the Snodar design as we could in software and used a minimum of hardware. This was primarily achieved by using a high reliability computer running GNU/Linux (Debian etch, with a read-only root filesystem) and having an external USB soundcard for analog IO. Fig 2 shows a high-level system overview of Snodar. The system consists of a remote (cold, $\left.-70^{\circ} \mathrm{C}\right)$ external antenna and an internal (warm, $-10^{\circ} \mathrm{C}$ ) data acquisition system.

It is straightforward to show that the power received from an acoustic radar as a function of scattering cross section, $\sigma$, and height, $h$, is

$$
P_{r}=\eta_{r} P_{t} \eta_{t} \sigma(\theta) \frac{c \tau}{2} A_{r} \frac{1}{h^{2}} e^{-2 \alpha h}
$$

where $P_{r}$ and $P_{t}$ are the power received and transmitted respectively, $c$ is the speed of sound, $\tau$ is the pulse length, $\alpha$ is the atmospheric attenuation coefficient and $\eta_{r}$ and $\eta_{t}$ are the receiver and transmitter efficiencies respectively. For an acoustic wave propagating in a turbulent atmosphere, the scattering cross section as a function of scattering angle, $\theta$, is given $b y^{6}$ 


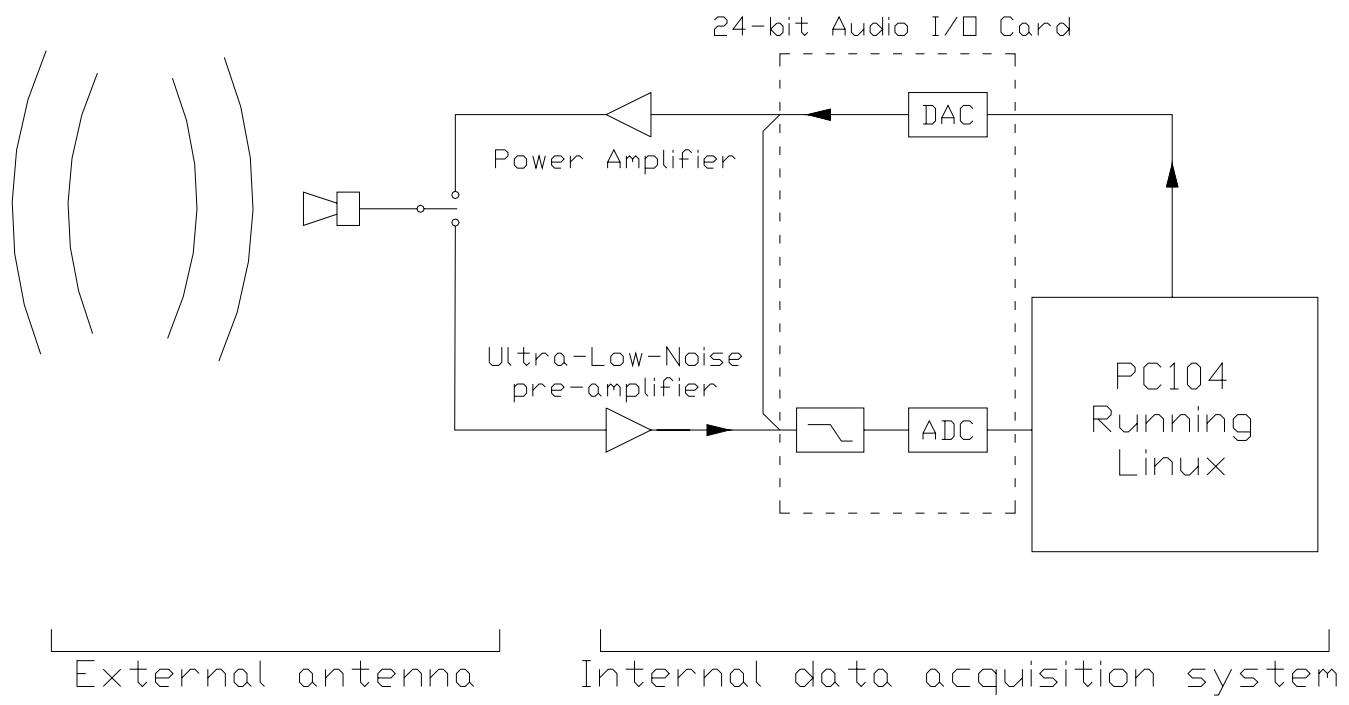

Fig 2 High level overview of Snodar. The system is broken into two parts, the cold external components and the warm internal components. All analogue IO is performed with a USB soundcard. Note the hardware loop back from the output to the input of the soundcard allowing IO synchronisation.

$$
\sigma(\theta)=0.03 \kappa^{1 / 3} \cos ^{2} \theta\left[\frac{C_{V}^{2}}{c^{2}} \cos ^{2} \frac{\theta}{2}+0.13 \frac{C_{T}^{2}}{T^{2}}\right] \sin \left(\frac{\theta}{2}\right)^{-11 / 3}
$$

where $\kappa$ if the wave vector, and $C_{T}^{2}$ and $C_{V}^{2}$ are the temperature and velocity structure function constants respectively. By locating the transmitter and receiver at the same position, i.e. making the instrument monostatic, the scattering angle becomes $\pi$ and equation (2) simplifies to

$$
\sigma_{\pi}=0.0039 \kappa^{1 / 3}\left[\frac{C_{T}^{2}}{T^{2}}\right]
$$

Making Snodar monostatic allows $C_{T}^{2}$ to be separated from $C_{V}^{2}$, however it also increases the problem of acoustic reverberation and ringing. This typically leads to an increase in the minimum sampling height of an acoustic radar.

Due to the extreme cold and dryness on the Antarctic plateau high frequency acoustic waves propagate rather well. The optimum operating frequency for Snodar is a function of acoustic scattering, atmospheric absorption and background acoustic noise. While the spectrum of the background acoustic noise is unknown, the optimum frequency in terms of acoustic scattering and atmospheric back scatter was found to be $5 \mathrm{kHz}$. An operating frequency of $5 \mathrm{kHz}$ equates to a pulse of just 33 cycles for $1 \mathrm{~m}$ resolution at an air temperature of $-40^{\circ} \mathrm{C}$. Snodar was designed to operate at this frequency.

Snodar operates from a single 28 VDC supply with an average power consumption of under $15 \mathrm{~W}$. All signal processing and control functions are performed by a PC/104 running GNU/Linux. The computer system is based on a read-only file system stored on USB flash drives for reliability reasons. When the system boots, the parts of the filesystem that need to be read-write (e..g, /etc, /var, /tmp/, /home) are loaded into a RAM disk and the system runs as normal. Since flash drives deteriorate after many write cycles, data are buffered on the RAM disk and periodically written to the flash drive to minimise write cycles and increase reliability. A Sound Blaster Live! 24-bit external USB sound card is used for all analog signal IO. The USB soundcard allows stereo signal capture at 24-bit resolution while sampling at $96 \mathrm{kHz}$ with a USB 2.0 connection. However, with a SNR of 100dB, Snodar only uses the top 16-bits. The Sound Blaster Live! 24-bit external USB sound card was used primarily because it offers an adequate sampling rate and SNR, and is also supported by the Advanced Linux Sound Architecture (ALSA) drivers. This means all IO is performed through the standard ALSA API calls, which provide hardware abstraction and greatly simplifies the software. 
After testing several acoustic transducers, the JBL2402H from JBL Professional series was selected. The JBL2402H is a horn-loaded compression driver (or "bullet tweeter") and has a frequency response from $2.5 \mathrm{kHz}$ to $15 \mathrm{kHz}$. The high operating frequency allows sharp pulses to be formed with little ringing. An ultra-low noise preamplifier is used to amplify the faint echoes from atmospheric scattering to a usable level. To increase the transducer's dampening and further reduce the ringing, the transducer is coupled to the preamplifier with an impedance-matching transformer. The transformer is wound on a shielded toroidal ferrite core and is housed at the transducer. An aerospace grade electromechanical relay, specified to operate at temperatures down to $-70^{\circ} \mathrm{C}$, is used to switch the transducer between the power amplifier output and the preamplifier input. This relay appears to have failed in February 2008, as mentioned in section 6 below.

A mono channel $60 \mathrm{~W}$ audio power amplifier is used to amplify the audio output of the USB soundcard. The power amplifier, a Redback A6040, can be powered from 28 VDC or 240 VAC. As the JBL2402H is rated for a maximum continuous power of $40 \mathrm{~W}$; the gain on the $\mathrm{A} 6040$ is set to limit the output voltage of the amplifier to $18 \mathrm{~V}_{\mathrm{RMS}}$ in order to ensure that the JBL2402H is not damaged. As the A6040 has a differential input, both channels of the stereo output from the USB soundcard are used. The stereo channels are driven in a pseudo-differential manner from software. Not only does this reduce the systems susceptibility to noise, but it also allows two devices to be driven from the same output. In this particular case, the power amplifier is driven by the difference of the two stereo channels, and the relay switching circuit is controlled by the average of the two channels. Again, this reduces the system complexity as all low-latency IO is handled by the ALSA drivers.

The transducer is mounted at the focal point of a $1.5 \mathrm{~m} \mathrm{f} / 0.6$ off-axis parabolic reflector-a commercial satellite TV dish. The transducer's radiation pattern is matched to the parabolic reflector f-ratio and results in a final beam width of approximately $4^{\circ}$. A $2.4 \mathrm{~m}$ tall sound cone is used to reduce acoustic background noise and fixed echoes. The sound cone is constructed in a modular fashion using 24 panels for easy shipping. The panels are made from $12 \mathrm{~mm}$ mediumdensity-fibre which has highly desirable acoustic properties. Each panel is upholstered with $40 \mathrm{~mm}$ of a rockwool based sound absorber to reduce acoustic reverberation within the cone. The rockwool based sound absorber is used as it has superior performance at extremely low temperatures when compared to polymer based materials. Electric heat pads have been attached to the underneath of the parabolic reflector to stop the formation of frost and sublimate small portions of snow. The electric heat pads are able to dissipate up to $200 \mathrm{~W}$ of power. Construction of the parabolic reflector and sound cone is shown in Fig 3.

\section{DATA PROCESSING}

As Snodar will operate in remote parts of Antarctica with limited, or no, communications, all data must be stored locally. If an echo is recorded every 30 seconds the compressed time-series data are of the order of $1 \mathrm{~GB}$ per day. While it is possible to store this volume of data locally, it is inconvenient and expensive. On-site data reduction is performed in real time to overcome this. The on-site data processing passes the signal through a band-pass filter centred at the operating frequency with a bandwidth of typically $500 \mathrm{~Hz}$. The average power per range bin is calculated from the filtered signal. While dependant on the sampling parameters, this simple on-site data processing typically reduces the amount of data to be stored by a factor of 600 . The reduced data are then post-processed off-site to correct for temperature gradients, noise and atmospheric attenuation. All post processing of the raw and pre-processed Snodar data is performed using MatLab.

Knowing the properties of the background noise is useful when designing the signal processing algorithms and selecting the optimum operating frequency of the instrument. For these reasons Snodar records the background noise before it transmits a pulse. The average noise power or raw time series can also be saved for further analysis. Each echo generates a single data file that is buffered on the RAM disk whether it be a raw time series or pre-processed data. Each data file contains the system state, recorded noise and the echo data. This allows the sampling conditions to be reconstructed from a single file. Once a specified amount of data has been buffered on the RAM disk it is compressed and written to the flash drive. The entire flash disk is retrieved the following summer. Small amounts of data can be downloaded via the Iridium satellite through UNSW's PLATO facility. 


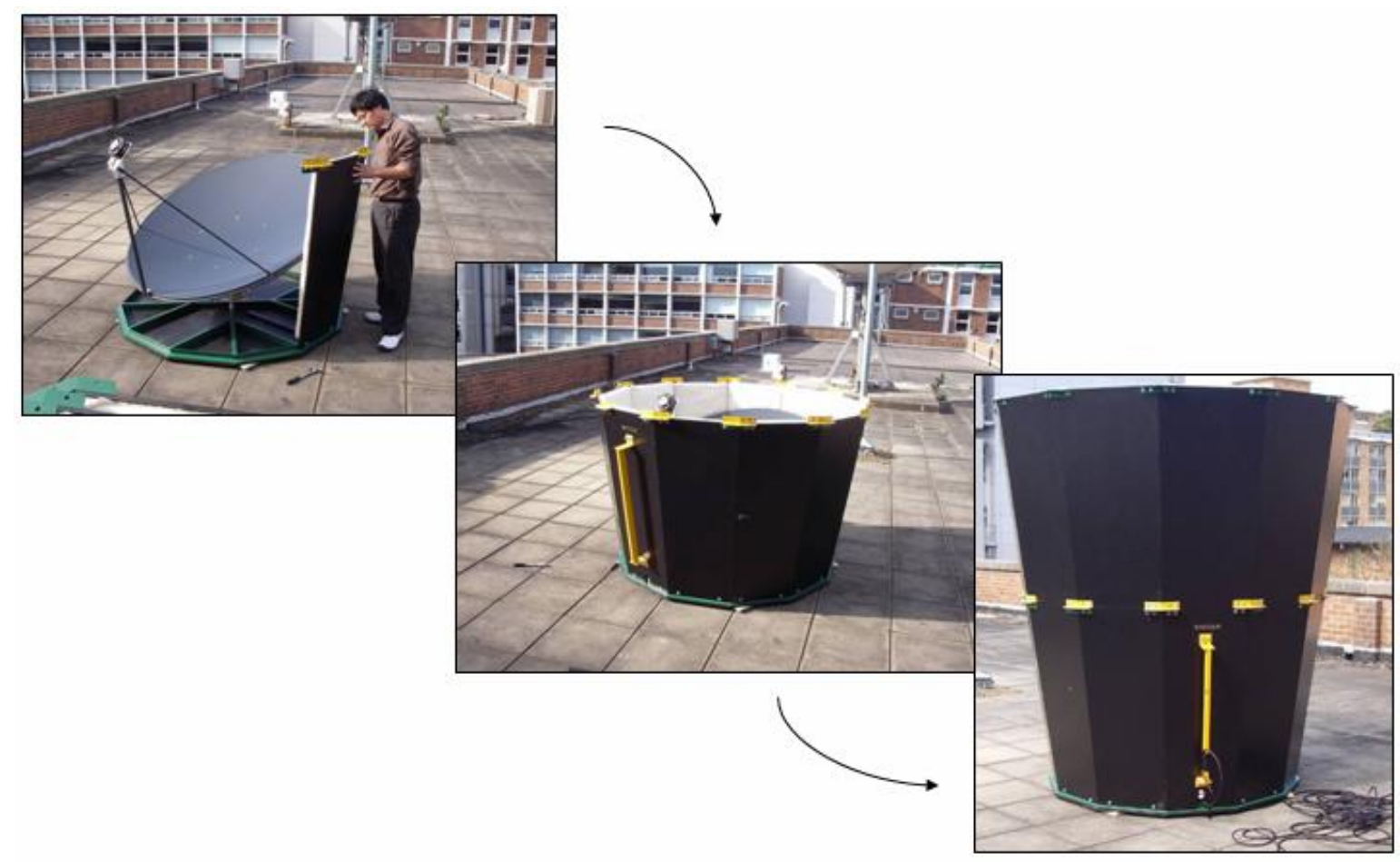

Fig 3 Left: The parabolic reflector mounted on the base plate with the transducer installed at its focus. Middle: First layer of panels installed. Right: The fully constructed sound cone.

\section{CALIBRATION}

Acoustic radars used in atmospheric research are typically nontrivial to calibrate when the absolute value of the scattering coefficient is required. As the height of the ABL can be defined through relative changes in $C_{T}^{2}$, absolute calibration is seldom required. However, if the temperature, pressure and $C_{T}^{2}$ profiles are known then the refractive index structure function constant $\left(C_{N}{ }^{2}\right)$ can be derived and its contribution to the astronomical "seeing" calculated. For these reasons it is desirable, but not essential, to calibrate Snodar. The fact that Snodar must operate over a $40^{\circ} \mathrm{C}$ temperature range, unmanned for a year posses an issue with the stability of the instrumental sensitivity. In an attempt to overcome this, we suspended a sphere of $50 \mathrm{~mm}$ diameter made from solid phenolic resin over the aperture of the sound cone. The sphere is referred to as the "calibration sphere" and has two purposes. Firstly, as the height of the calibration sphere above the ice surface is accurately known the echo can be used to remove any systematic offset errors in the scattering heights. Secondly, if Snodar operates at a constant wavelength then the magnitude of the echo from the calibration sphere should be constant throughout the year. This will allow the efficiency of the system to be monitored and the transducer sensitivity to be corrected during post processing.

The process of calibration is typically done at several heights against a fully instrumented tower. Microthermal sensors, or differential thermocouples, were mounted on a $6 \mathrm{~m}$ tower and used to calibrate Snodar. Snodar was set up next to the tower and simultaneous measurements were made with both instruments. Unfortunately, there were fixed echoes from ground clutter in the Snodar data, however, after examining the data it appeared that the fixed echoes at the height of the microthermals were comparable to the echo from atmospheric scattering and there was indeed a correlation between the values of $C_{T}^{2}$ given by the microthermal and Snodar. As the power from fixed echoes is comparable to the atmospheric scattering it is possible for the power from fixed echoes to be estimated and removed as follows:

$$
\sigma_{\pi}=\frac{\int P_{R}}{\int P_{T}}=\frac{\int P_{A T M}+\int P_{F I X E D}}{\int P_{T}}=\frac{\int P_{A T M}}{\int P_{T}}+\sigma_{F I X E D}
$$


where $P_{A T M}$ and $P_{F I X E D}$ are the power from atmospheric scattering and fixed echoes respectively. The reasonable assumption was made that at some point $P_{A T M}$ will be much less then $P_{F I X E D}$ and $\sigma_{\pi}$ will be approximately $\sigma_{F I X E D}$. Therefore $\sigma_{F I X E D}$ was estimated to be the smallest value recorded by Snodar throughout the dataset. A least-squares fit was then used to calibrate Snodar from the microthermal measurements after the microthermal were calibrated in a laboratory. Fig 4 shows the calibrated measurements and the correlation between Snodar and the microthermal. The error from this simple calibration is a factor of three, giving an order of magnitude estimate for $C_{T}^{2}$.
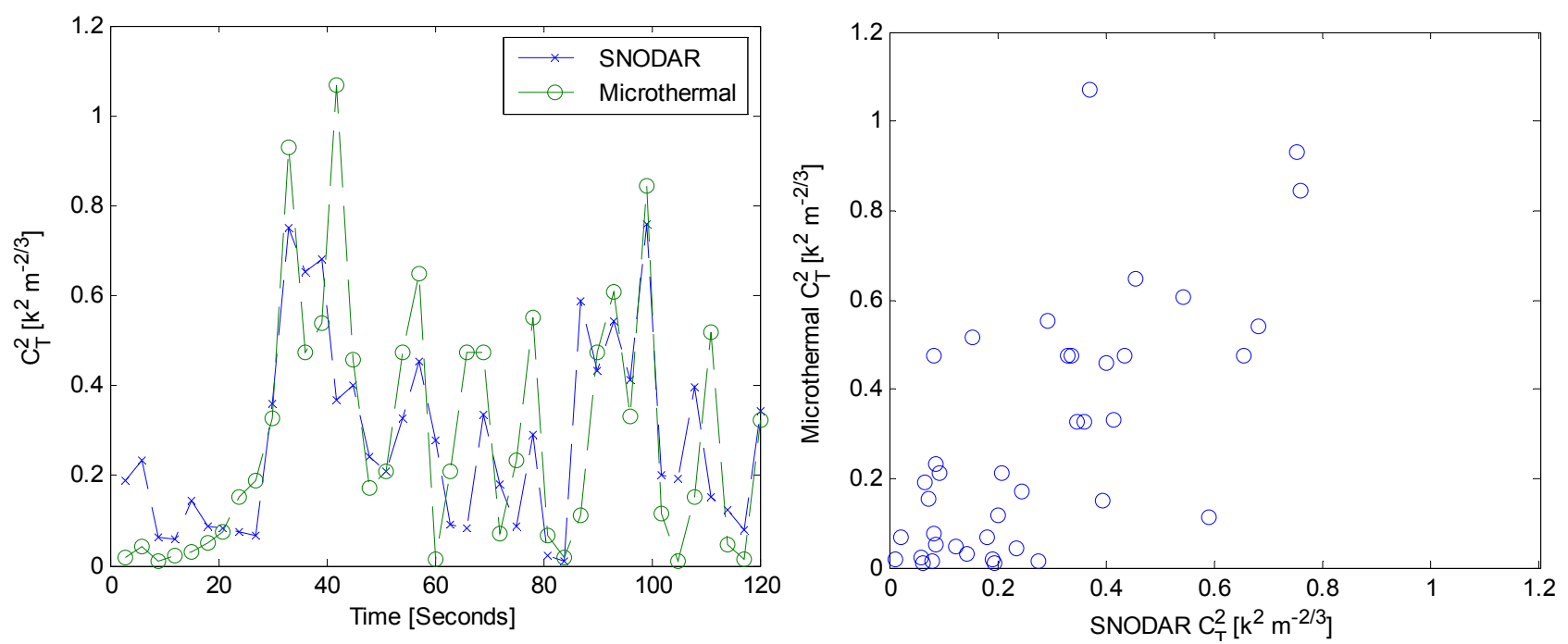

Fig 4 Calibrated Snodar and microthermal measurements. Left: $C_{T}^{2}$ given by Snodar and the microthermal as a function of time. There was one sample every 3 seconds with a total of 40 samples. Right: Correlation of Snodar and microthermal measurements.

\section{INITIAL RESULTS}

As Snodar was designed to operate at high frequencies the atmospheric attenuation of $5 \mathrm{kHz}$ makes it difficult to fully test in temperate climates. The functionality and operation of the instrument was confirmed in Sydney, Australia and underwent a small commissioning period once installed at Dome A by the Polar Research Institute of China PANDA traverse. After setting the gain to the appropriate level, Snodar started to collect data. Fig 5 shows a single echo from Snodar while operating at Dome A at a frequency of $5 \mathrm{kHz}$ with $1 \mathrm{~m}$ resolution. Fig 5 shows the time series data after digital filtering as well as the result of the pre-processing algorithm. The height scale has not been corrected for temperature gradients in these figures.

\section{FURTHER WORK}

Snodar was deployed to Dome A in January 2008 and started collecting data after a small commissioning period. Various sampling schemes were used over several weeks, however, Snodar then failed to detect echoes, and it is suspected that the electromechanical relay failed. This means that Snodar is no longer able to transmit pulses. It can however, monitor the acoustic background noise. There is a substantial amount of work to be done in the post processing and analysis of the data that was gathered. Various modifications to Snodar are currently being designed in order to increase reliability. The electromechanical relay has been replaced with a solid state solution that also replaces the audio power amplifier with an efficient switching network. Whilst heating of the parabolic reflector reduces icing, it does however require a large amount of power which is not always available. Various geometric designs, membrane covers and other heating methods are currently being investigated to overcome icing issues. 


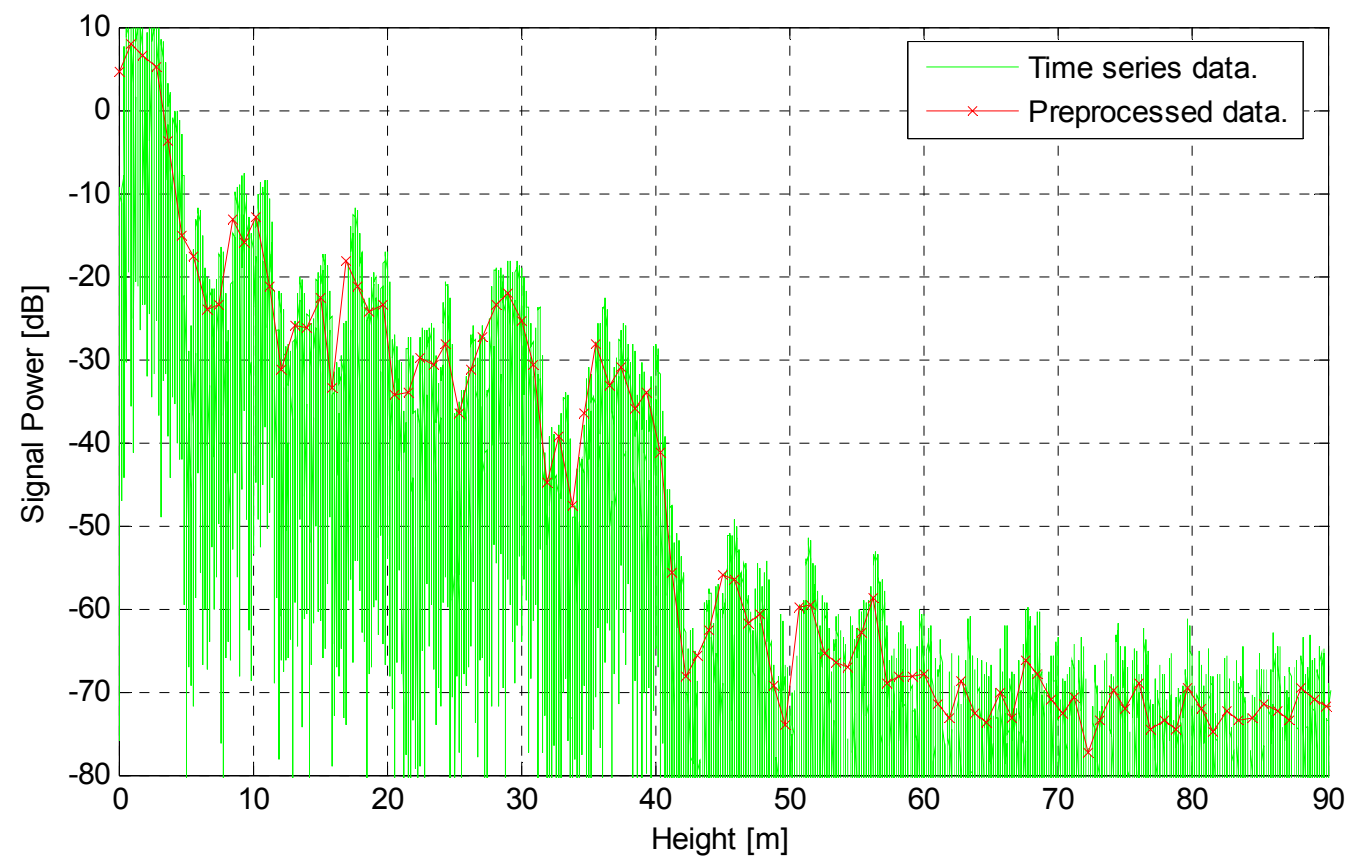

Fig 5 A single echo from Snodar while operating at $5 \mathrm{kHz}$ with $1 \mathrm{~m}$ resolution. The green line is the time series signal after digital filtering. The red line is the result of the on-site preprocessing algorithim which calculates the average power per range bin.

\section{ACKNOWLEDGEMENTS}

The authors thank Kiera Lindsay for her invaluable help upholstering the sound absorbing material to the inside of Snodar's sound cone, Jon Everett for procurement and finally the entire Polar Research Institute of China PANDA traverse team, with particular mention of Xu Zhou and Zhenxi Zhu, for installing and commissioning Snodar at Dome A. This research is financially supported by the University of New South Wales and the Australian Research Council. We also thank Elena Masciadri for supplying the thermocouples that were used to calibrate Snodar under the Marie Curie Excellence Grant (FOROT) MEXT-CT-2005-023878.

\section{REFERENCES}

[1] Lawrence, J.S., Ashley, M.C.B., Tokovinin, A., Travouillon, T., "Exceptional astronomical seeing conditions above Dome C in Antarctica", Nature, 431, 278-281 (2004).

[2] Agabi, A., Aristidi, E., Azouit, M., Fossat, E., Martin, F., Sadibekova, T., Vernin, J., Ziad, A., "First Whole Atmosphere Nighttime Seeing Measurements at Dome C, Antarctica", The Publications of the Astronomical Society of the Pacific, 118, 344-348 (2006).

[3] Swain, M.R., Gallée, H., "Antarctic Boundary Layer Seeing", The Publications of the Astronomical Society of the Pacific, 118, 1190-1197 (2006).

[4] Lawrence, J.S., Allen, G., Ashley, M.C.B., et al. "The PLATO Antarctic site testing observatory”, Proc SPIE, this proceedings (2008).

[5] Little, C.G., "Acoustic methods for the remote probing of the lower atmosphere", Proceedings of the IEEE, 57, 571578 (1969).

[6] Tatarskii, V., [The effects of the turbulent atmosphere on wave propagation], Keter Press, Jerusalem (1971). 